\title{
A Bright Deep-Red AlEgen with Locked Noncoplanar Conformation for Lipid Droplet-Specific Imaging
}

\author{
Ying Yu, ${ }^{1, \dagger}$ Hao Xing,,${ }^{1,}$ Hojeong Park, ${ }^{1}$ Ruoyao Zhang, ${ }^{1}$ Chen Peng, ${ }^{1}$ Herman H.-Y. Sung, ${ }^{1}$ Ian D. Wil- \\ liams, ${ }^{1}$ Chao Ma, ${ }^{3}$ Kam Sing Wong, ${ }^{3}$ Min-Hui Li, ${ }^{4}$ Zheng Zhao, ${ }^{2 *}$ Ben Zhong Tang ${ }^{1 *}$ \\ ${ }^{1}$ Department of Chemistry, Hong Kong Branch of Chinese National Engineering Research Center for Tissue Restoration and \\ Reconstruction and Institute for Advanced Study, Department of Chemical and Biological Engineering, Division of Life Sci- \\ ence and State Key Laboratory of Molecular Neuroscience, The Hong Kong University of Science and Technology, Clear \\ Water Bay, Kowloon, Hong Kong, China. E-mail: tangbenz@ust.hk \\ ${ }^{2}$ School of Chemistry and Chemical Engineering, Southeast University, Nanjing, China. E-mail: zhaozheng@seu.edu.cn \\ ${ }^{3}$ Department of Physics, The Hong Kong University of Science and Technology, Clear Water Bay, Kowloon, Hong Kong, \\ China \\ ${ }^{4}$ Chimie ParisTech, PSL University Paris, CNRS, Institut de Recherche de Chimie Paris, 75005 Paris, France
}

\begin{abstract}
In the field of fluorescent materials, bright deep-red/near-infrared (DR/NIR) luminogens are always in demand whose rationally and accurately design yet are challenging. Current design strategy of DR/NIR emitters mainly depends on the construction of large $\pi$-conjugation and strong donor-acceptor system, which commonly suffers the quenching factors from $\pi-\pi$ stacking and twisted intramolecular charge transfer (TICT) effect. In this work, we employed the non-covalent interactions and constructed a luminogen with rigid and twisted conformation, which not only red-shifts the emission wavelength of the luminogen to DR/NIR region but also effectively suppresses the non-radiative decay by $\pi-\pi$ stacking and TICT. Moreover, the rational molecular design endows the new luminogen with two-photo absorption capability and superior selectivity towards lipid droplets in different levels including living cells, tissues, and live fish embryo. This work thus presents an elegant, facile and efficient strategy for generating functional DR/NIR emitters.
\end{abstract}

\section{INTRODUCTION}

It is generally believed that the properties of the molecules are determined by their structures, which includes the constitution, configuration and conformation. In most cases, the constitution and configuration are difficult to change once being selected during the preparation process. While conformation, representing the spatial arrangement of the atoms affording distinction which can be interconverted by rotations about formally single bonds, is more flexible. ${ }^{1}$ For example, a cyclohexane conformation is any of several three-dimensional shapes adopted by a cyclohexane molecule. But these shapes can never be separated due to the fast ring flip. When the energy barriers between different conformations are large enough, they can be isolated through multiple preparation methods, taking 2,2',6,6'--tetrasubstituted biphenyls as an example. ${ }^{2}$ Different conformations usually exhibit distinct properties reflecting the fundamental structure-property relationship. ${ }^{3,4}$ Therefore, precisely tuning the conformation is highly desirable but exceptionally challenging based on the fact that "easy to change" can also be described as "hard to control".,

Among those properties that are strictly related to conformation, ${ }^{7,8}$ fluorescence holds its unique features such as low energy consumption, stimuli responsiveness, and non-invasiveness exhibiting broad applications in electroluminescence devices, ${ }^{9,10}$ chemo/bio-sensors, ${ }^{11,12}$ and bioimaging etc. ${ }^{13,14}$ The luminescence property of a single molecule generally relies on the excited state features such as energy level distribution, intersystem crossing, and exciton decay pathway etc. according to the Jablonski diagram. Typically, these features correlate closely with the molecular conformation. The conformational torsion and conformation rigidity not only influence the energy level distribution of the molecule but also affect the radiative/nonradiative decay rate of the exciton. ${ }^{15,16}$ Overall, flexible conformation will cause the emission wavelength redshift and luminescence efficiency decrease, while rigid conformation will suppress the non-radiative decay and thus alleviate the luminescence efficiency. ${ }^{17-19}$ Despite lots of strategies have been proposed to prepare luminescent materials with diverse colors and efficiency, ${ }^{20-23}$ it is still challenging to design efficient deep red/near-infrared (DR/NIR) luminogens $(650-1300 \mathrm{~nm})$ since non-radiative decay is generally more competitive to radiative decay in this wavelength region due to the intrinsic energy gap law and some fluorescence quenching factors like twisted intramolecular charge transfer (TICT) and strong intermolecular interactions etc. ${ }^{24-26}$ In this regard, designing luminescent materials by conformation control possibly helps to improve the luminescence efficiency of DR/NIR luminogens because both strong intermolecular interaction and structure rigidity could be tuned by conformation control to facilitate the luminescence efficiency. ${ }^{27,28}$ Therefore, conformation control strategy possibly is a promising strategy to develop efficient DR/NIR luminogens. 
A

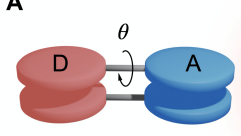

$\theta=0^{\circ}$
B

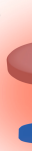

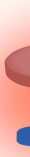

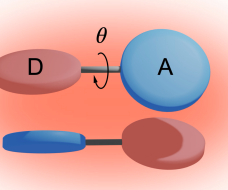

$0^{\circ}<\theta<90^{\circ}$
C

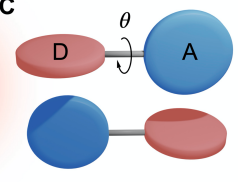

$\theta=90^{\circ}$
Figure 1. Cartoon representation of three cases, the dihedral angle between donor and acceptor moieties is (A) $0^{\circ}$, (B) $0^{\circ}<\theta<90^{\circ}$, and (c) $90^{\circ}$.

Organic DR/NIR luminogens are in great demand due to their extensive applications in flexible optoelectronics and bio-medical fields. ${ }^{29-31}$ Constructing donor-acceptor (D-A) structure is the commonly utilized strategy to prepare DR/NIR luminogens, in which the torsion between the D and A moieties plays a significant role in determining the photophysical properties of the molecule (Figure 1). The dihedral angle $(\theta)$ between the $\mathrm{D}$ and A moieties theoretically has three cases: $0^{\circ}, 0^{\circ}-90^{\circ}$, and $90^{\circ}$. When $\theta=0^{\circ}$, the coplanar structure enjoys a favorable conjugation, which benefits the electron delocalization and the allowed transition. However, the close packing and infinite $\pi-\pi$ stacking will quench the fluorescence in the aggregated or solid state due to excessive energy splitting and strong vibrational coupling between the ground state and the excited state, which is a fatal problem for conventional fluorophores, named aggregation caused quenching (ACQ).$^{32}$ When $\theta=90^{\circ}$, the twisted structure suppresses the ACQ effect by avoiding strong $\pi-\pi$ stacking, but the emission usually exhibits a wavelength redshift followed by a quantum yield (QY) decrease due to the TICT effect. ${ }^{33-35}$ The low QY is because the strong charge separation in TICT state will cause a forbidden transition and is easy to undergo the non-radiative conical intersection to quench the emission. ${ }^{35}$ In real cases, $\theta$ regularly locates in between $0^{\circ}-$ $90^{\circ}$ rather than the extreme $0^{\circ}$ or $90^{\circ}$. Since a small $\theta$ close to $0^{\circ}$ easily causes the ACQ effect, it is more common to design a molecule with a twisted structure to maintain the solid-state emission. Although the TICT strategy is effective in red-shifting the emission wavelength, it is unavoidable to sacrifice parts of the QY, resulting in the dim luminescence. In this regard, if any chemical or physical restriction factors could lock the noncoplanar conformation, the antagonism between the emission red-shift and QY decrease may be solved elegantly, affording luminogens with both long emission wavelength and high QY. Noting that although chemical linking has been utilized to fix the molecular conformation, the tedious synthesis somehow limits the real implement. ${ }^{36,37}$ Therefore, a more facile and manipulatable strategy that can immobilize the molecular conformation should be explored in tuning the luminescence of the emitters.

Non-covalent interactions such as hydrogen bonding, ${ }^{38,39}$ electrostatic interactions, ${ }^{40,41}$ halogen bonding, ${ }^{42,43}$ and hostgust interactions $\mathrm{s}^{44,45}$ have been well explored in the construction of advanced functional materials. Among them, intra/intermolecular interactions have exhibited their unique functionalities. For example, Zhu et al. found that the intramolecular hydrogen bonding helps keep the linear ribbon structure of the diketopyrrolopyrrole-based semiconductors, which benefits the efficient charge transport. ${ }^{46}$ Jiang and co-workers designed a series of two-dimensional covalent organic frameworks locked by intralayer hydrogen-bonding, which suppresses the torsion of the edge units and locks the tetragonal sheets in a planar conformation. ${ }^{47}$ These pioneering works have inspired the research of hydrogen bonding as a powerful tool to fine-tuning the conformation and assembly behavior of molecular materials. However, employing hydrogen bonding to rigidify the molecular conformation and to develop DR/NIR luminescent materials have been rarely investigated, which possibly motivate the new design of efficient DR/NIR luminogens. ${ }^{48}$

In this work, we have developed a new fluorine-containing acceptor which could react with triphenylamine to construct a distinct D-A molecule 2-(4-(4-(diphenylamino)phenyl)-5fluoro-[1,3]dithiolo[4',5':3,4]benzo[1,2c][1,2,5]thiadiazol-7ylidene)malononitrile (TPABSM). TPABSM shows a DR emission whose emission spectrum covers from visible to NIR I region with a high quantum yield of $13 \%$. Our results indicate that both intra and intermolecular hydrogen bonding help rigidify the torsional conformation of TPABSM, which not only shifts the emission to DR region but also suppresses the excited state molecular motion and the resulted non-radiative decay, affording the highly bright DR emission of TPABSM. Furthermore, TPABSM exhibits excellent two-photon imaging and lipid droplet targeting capabilities, facilitating its application in high-resolution fat tissue imaging and in vivo imaging of yolk sac in zebrafish embryos.

\section{RESULTS AND DISCUSSION}
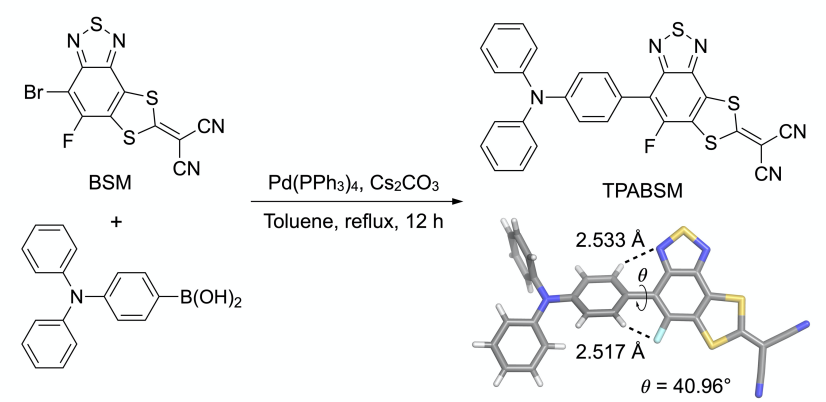

Figure 2. Synthetic route to TPABSM and its single crystal structure.

The synthetic route to TPABSM was presented in Figure 2 and Figure S1. Nucleophilic substitution between 4,7-dibromo5,6-difluorobenzo[c] $] 1,2,5]$ thiadiazole and sodium 2,2-dicyanoethene-1,1-bis(thiolate) produces the intermediate of monodisulfovalene fused benzodithiazole (BSM) with a dual-disulfovalene fused benzodithiazole (BDSM) as a byproduct. Suzuki coupling of BSM and 4-(diphenylamino)phenylboronic acid gives the final product TPABSM with a yield of $58 \%$. The intermediates and final products were fully characterized by ${ }^{1} \mathrm{H}$, ${ }^{13} \mathrm{C},{ }^{19} \mathrm{~F}$ NMR and high-resolution mass spectra (Figures S2-S7). The single crystals of BDSM and TPABSM were obtained through the solvent evaporating method, which further confirmed the reaction procedure and the structure of targeting product (Figure 2, Figure S8 and Tables S1-S2). The single crystal structure of TPABSM shows that the torsional angle between the TPA moiety and BSM moiety is $40.96^{\circ}$. Furthermore, much shorter distances between the hydrogen atom on the phenyl ring and the fluorine $(2.517 \AA)$ and nitrogen $(2.533 \AA)$ atoms on BSM moiety suggest that strong intramolecular hydrogen bonding exist which is helpful to rigidify the molecular conformation. 

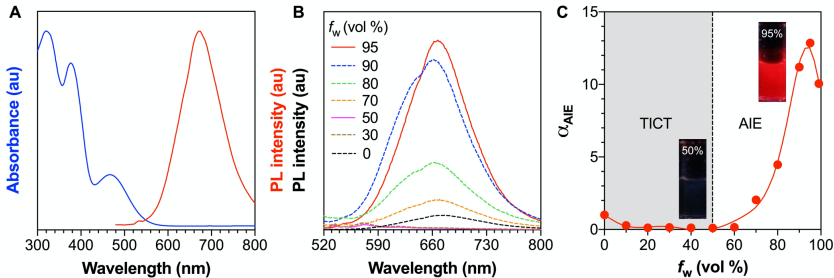

Figure 3. (A) Absorption and PL spectra of TPABSM in THF solution; (B) PL spectra of TPABSM in water/THF mixtures with different water fractions $\left(f_{\mathrm{w}}\right)$; (C) Plots of relative PL intensity $\left(\alpha_{\mathrm{AIE}}\right.$ $=I / I_{0}, I_{0}=$ PL intensity in pure THF at $\left.672 \mathrm{~nm}\right) v s f_{\mathrm{w}} . c=10 \mu \mathrm{M}$, $\lambda_{\mathrm{ex}}=460 \mathrm{~nm}$.

The photophysical properties of TPABSM were first examined. As shown in Figure 3A, the tetrahydrofuran (THF) solution of TPABSM exhibited three absorption peaks at $315 \mathrm{~nm}$, $370 \mathrm{~nm}$ and $460 \mathrm{~nm}$, in which the first one could be assigned to a $\pi-\pi^{*}$ absorption, the second one arose from the BSM moiety, and the last one was from the intramolecular charge transfer (ICT) absorption. ${ }^{49}$ TPABSM gave a much brighter red emission in toluene solution with an emission wavelength at $605 \mathrm{~nm}$ and a QY of $67.9 \%$. While in THF solution, the emission wavelength red-shifted to $669 \mathrm{~nm}$ with a QY of $4.1 \%$. The varied luminescence behavior of TPABSM in different solvents suggests the existence of TICT effect, which was further confirmed by the DFT calculation (Figure S9) and the solvatochromic results (Figure S10 and Table S3). Although the TICT effect is detrimental to the luminescence process, it could be suppressed in nonpolar toluene solution. More importantly, the intramolecular hydrogen bonding integrated with the TICT destabilization effect in toluene help to lock and immobilize the conformation of TPABSM in a more rigid form, resulting in the much higher QY. Intrinsically, this conformation rigidification enhanced luminescence is similar to the aggregation-induced emission (AIE) process, in which the conformation rigidification resulted by aggregation is thought to benefit the luminescence behavior. We thus investigated the luminescence behavior of TPABSM in the mixture of THF and water to see whether it is AIE-active. When adding water into the THF solution, the photoluminescence (PL) intensity decreased with the water fraction $\left(f_{\mathrm{w}}\right)$ in a range of $0-50 \%$. In this case, the TICT effect dominated the photophysical process and reduced the emission intensity. When the $f_{\mathrm{w}}$ exceeded $50 \%$, the TPABSM molecule began to aggregate and made the local microenvironment less polar. Therefore, the conformation rigidification became dominated and reversed the PL intensity from weak to strong. Thus, TPABSM exhibits typical AIE property. ${ }^{50,51}$
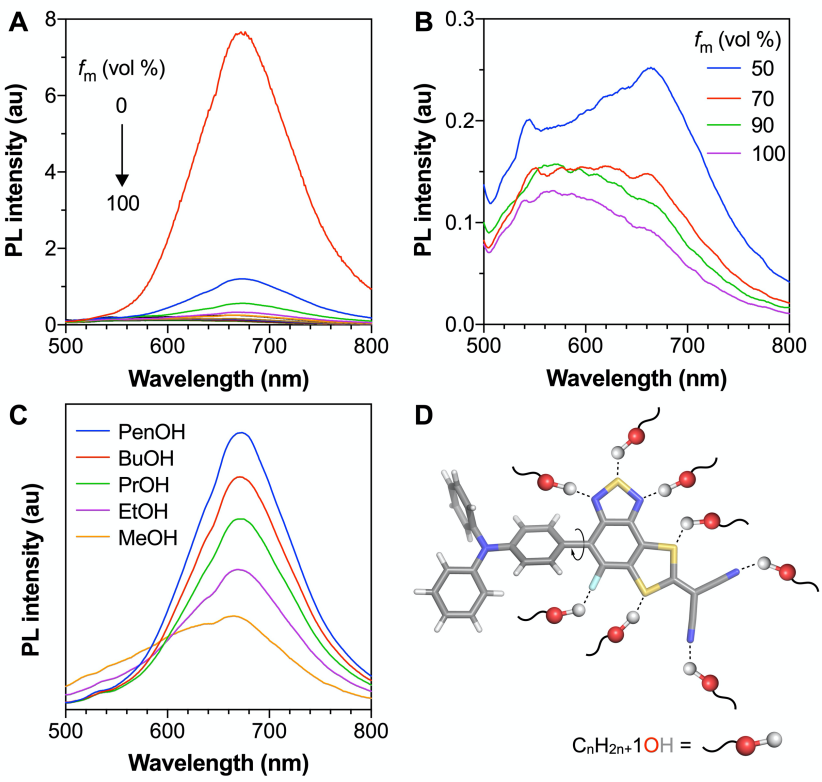

$\mathrm{C}_{n} \mathrm{H}_{2 n+1} \mathrm{OH}=\sim \mathrm{O}=\mathrm{O}$

Figure 4. (A) PL spectra of TPABSM in $\mathrm{MeOH} / \mathrm{THF}$ mixtures with different methanol fractions $\left(f_{\mathrm{m}}\right)$; (B) Amplified PL spectra in (A) with high methanol fractions; (C) PL spectra of TPABSM in alcohol/THF mixtures with alcohol fractions $\left(f_{\text {alcohol }}\right)=10 \%$; (D) Cartoon representation of the competitive solvent-solute H-bonds between alcohol and TPABSM. $c=10 \mu \mathrm{M}, \lambda_{\mathrm{ex}}=460 \mathrm{~nm}$.

To further elucidate the role of conformation rigidification in improving the emission property of TPABSM, the luminescence behavior of TPABSM in the mixture of THF and different alcohols was investigated. Theoretically, alcohols can work as hydrogen bonding donor to compete with the intramolecular hydrogen bonding interactions and thus influence the luminescence behavior. As shown in Figure 4A and 4B, when adding methanol into the THF solution of TPABSM, the emission intensity at around $669 \mathrm{~nm}$ gradually decreased. The amplified PL spectra of TPABSM showed that when the methanol fraction $\left(f_{\mathrm{m}}\right)$ exceeded $50 \%$, a new peak at around $520 \mathrm{~nm}$ emerged with the disappearance of the peak at $669 \mathrm{~nm}$. The peak at $520 \mathrm{~nm}$ overlapped well with the emission peak of BSM moiety, suggesting that the peak at $520 \mathrm{~nm}$ originates from the BSM moiety (Figure S11). These results indicate that the addition of methanol destroys the intramolecular hydrogen bonding and promotes the molecular twisting and the excited state molecular motion. As a result, the TICT state emission was quenched, and the LE state emission of BSM was retained.

Additionally, we also investigated the influence of different alcohols on the intramolecular hydrogen bonding. Figure 4C showed that in the presence of various alcohols, the PL intensity decrease was varied. The shorter alkyl chain of the alcohols, the weaker emission of the TPABSM solutions. This is because the size of the alcohols may interfere with the collision and interaction of the alcohol molecules with TPABSM molecules. In general, small methanol molecules is much easier to interact with the TPABSM molecules to form intermolecular hydrogen bonding that competes with the intramolecular hydrogen bonding, resulting in the PL decrease. The pentanol with a larger size is difficult to interact with the molecules of TPABSM due to the steric hindrance effect, resulting in the higher PL intensity of TPABSM in pentanol. Figure 4D gave the schematic diagram about how the alcohols destroy the intramolecular hydrogen bonding and accelerate the molecular motion to weaken the 
emission. The theoretical calculation also confirmed that there were evident non-covalent interactions between the benzodithiazole moiety and the adjacent phenyl ring (Figure S12). ${ }^{52,53}$

Since temperature change may influence the molecular motion $^{54,55}$ and thus change the molecular conformation as well as the microenvironment of the hydrogen atoms, variable-temperature ${ }^{1} \mathrm{H}$ NMR experiment was thus implemented to monitor the intramolecular hydrogen bonding change of TPABSM with temperature. As shown in Figure S13, with the temperature increasing, the chemical shift of the $\mathrm{H}$ atom that participates in the hydrogen bonding formation $\left(\mathrm{H}_{\mathrm{a}}\right)$ slightly shift to the higher magnetic field. As a contrast, the ${ }^{1} \mathrm{H}$ NMR signal of the $\mathrm{H}$ atom that without intramolecular hydrogen bonding $\left(\mathrm{H}_{\mathrm{b}}\right)$ shows a larger extent chemical shift. The smaller magnitude of the chemical shift of $\mathrm{H}_{\mathrm{a}}$ than $\mathrm{H}_{\mathrm{b}}$ suggests that the intramolecular hydrogen bonding benefits suppressing the molecular motion, which results in the smaller chemical shift change of $\mathrm{H}_{\mathrm{a}}$ than $\mathrm{H}_{\mathrm{b}}$.

TPABSM molecules also show bright emission in the solid state since the locked nonplanar conformation can avoid the strong intermolecular $\pi-\pi$ interactions. Indeed, no strong intermolecular $\pi-\pi$ stacking has been observed in the crystal packing of TPABSM. However, multiple intermolecular non-covalent interactions such as $\mathrm{S} \cdots \pi, \mathrm{C} \equiv \mathrm{N} \cdots \pi, \mathrm{C}-\mathrm{H} \cdots \mathrm{N}, \mathrm{C}-\mathrm{H} \cdots \mathrm{S}$, and intermolecular hydrogen bonding interactions were observed which helps lock the solid-state molecular motion (Figure S14). As a result, the solid of TPABSM exhibit much strong deep red emission at a wavelength of $670 \mathrm{~nm}$ with a quantum yield of $13 \%$.

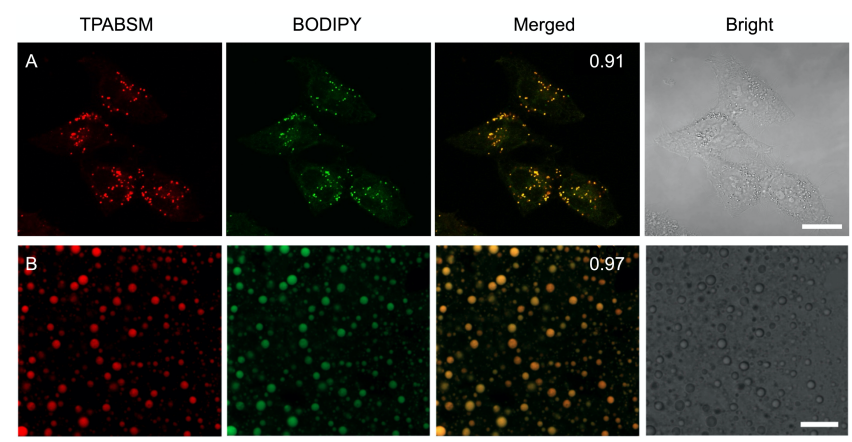

Figure 5. (A) Confocal fluorescent images of living HeLa cell stained with TPABSM $(2 \mu \mathrm{M}, 1 \mathrm{~h})$ and BODIPY $(2 \mu \mathrm{M}, 1 \mathrm{~h})$, scale bar $=20 \mu \mathrm{m}$; (B) Co-stained ex vivo confocal one-photon images TPABSM $(2 \mu \mathrm{M}, 1 \mathrm{~h})$ and BODIPY $(2 \mu \mathrm{M}, 1 \mathrm{~h})$ in high-fat feeding mice liver tissue, scale bar $=10 \mu \mathrm{m}$. TPABSM: $\lambda_{\mathrm{ex}}=488 \mathrm{~nm}, \lambda_{\mathrm{em}}$ $=550-650 \mathrm{~nm}$; BODIPY: $\lambda_{\mathrm{ex}}=488 \mathrm{~nm}, \lambda_{\mathrm{em}}=490-535 \mathrm{~nm}$. The number on the picture is co-localization coefficient.

Luminescent molecules with bright and deep-red emission generally are favorable in bio-imaging applications since they hold the advantages of non-invasiveness, deep penetration depth, superior anti-photobleaching capability, and low photodamage to cell or tissue. ${ }^{56,57}$ On the other hand, researchers have found that lipid droplets (LDs), used to be considered only as inert reservoirs of lipids for energy storage, have an essential influence on the normal function of cells. ${ }^{58}$ The abnormalities of LDs will lead to several like chronic hepatitis and liver cirrhosis etc. Therefore, the development of superior LDs probe and study the physiological behavior of LDs become highly significant in biomedical research. ${ }^{59,60}$ TPABSM is a superior liposoluble luminescent molecule, and the fluorine is a commonly used atom to improve the lipophilicity of drug molecules, ${ }^{61,62}$ which thus inspired us to explore the possibility of TPABSM as a dye for LDs imaging. Before the experiment, we first calculated the $\log \mathrm{P}$ (n-octanol/water partition coefficient) value of TPABSM because pioneering work has reported that organic dyes for lipid droplets staining usually exhibited $\log P$ value larger than 5 predicted by using QSAR (quantitative structure activity relations) models. ${ }^{63,64}$ The calculated $\log \mathrm{P}(\mathrm{Clog} \mathrm{P})$ value of TPABSM is as high as 8.403 which was much higher than those LDs specific dyes like Nile Red (4.618) and BODIPY 493/503 (5.028), ${ }^{63}$ suggesting TPABSM may be a suitable dye for LDs imaging. As shown in Figures S15 and S16, incubated with $2 \mu \mathrm{M}$ TPABSM for $1 \mathrm{~h}$, the A549 and Hela cells were successfully stained and emitted around $640 \mathrm{~nm}$, suggesting a fast permeability of TPABSM for living cells. To confirm the specificity of the TPABSM for LDs imaging, the co-localization experiments were then carried out by incubating Hela cells with TPABSM and BODIPY 493/503, a commercial dye for LDs. The results showed that TPABSM stains LDs specifically with a co-localization coefficient of 0.91 (Figure 5A). Cell viability is an important parameter for bioimaging application. The cytotoxicity was evaluated using 3-(4,5-dimethyl-2-thiazolyl)-2,5-diphenyltetrazolium bromide (MTT) assay under different TPABSM concentrations. As suggested in Figure S17, no significant variation in cell viability was observed, indicating that TPABSM possesses good cell biocompatibility. Moreover, TPABSM showed better photostability due to its AIE nature (Figure S18).

Apart from living cells, the LDs of liver tissues of mice can also be clearly stained by TPABSM, suggesting the high penetration depth. By comparing the images of normal and high-fat feeding mice liver tissues, apparent deep-red emission was observed in high-fat feeding mice liver tissue (Figure S19). Furthermore, co-staining experiments with BODIPY shows a high co-localization coefficient $(0.97)$, indicating the potential of TPABSM for the specific tissue or in vivo LDs imaging (Figure 5B).

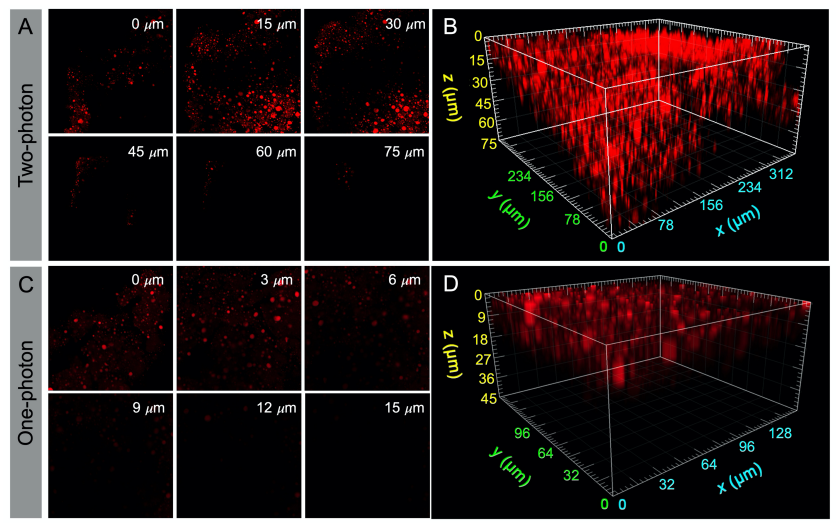

Figure 6. Ex vivo fatty liver tissue imaging. (A) Two-photon ( $\lambda \mathrm{ex}$ $=880 \mathrm{~nm})$ images of and $(\mathrm{C})$ one-photon $\left(\lambda_{\mathrm{ex}}=488 \mathrm{~nm}\right)$ images of high-fat feeding mice liver tissue stained with TPABSM $(4 \mu \mathrm{M}, 1$ h) at different penetration depths. $\lambda_{\mathrm{em}}=550-650 \mathrm{~nm}$ (B) Reconstructed 3D two-photon images. (D) Reconstructed 3D onephoton images. Scale bar: $40 \mu \mathrm{m}$ in (A) and $20 \mu \mathrm{m}$ in (C). Twophoton penetration depth: $72 \mu \mathrm{m}$, One-photon penetration depth: $15 \mu \mathrm{m}$.

Although TPABSM exhibited a long emission wavelength $(670 \mathrm{~nm})$ and high QY, its short absorption wavelength $(<500$ $\mathrm{nm}$ ) suffer the drawbacks of auto-fluorescence, tissue or skin scattering which limits its application for deeper tissue or in vivo imaging. On the other hand, in comparison with 
luminogens with one-photo excitation, the excitation wavelength of two-photon luminogens can be extended to a deep red region or even near-infrared region, which can successfully overcome the drawbacks of short wavelength excitation. ${ }^{65-67}$ Two-photon absorption (2PA) cross-section $\left(\delta_{2 \mathrm{PA}}\right)$ is a crucial parameter for two-photon excitation. The 2PA of TPABSM was studied using a two-photon excited fluorescence (TPEF) technique with a femtosecond pulsed laser source, and the relative TPEF intensity was measured using Rhodamine B as the standards. The maximum $\delta_{2 \mathrm{PA}}$ value determined at $880 \mathrm{~nm}$ is as high as 107.6 GM (Figure S20). In comparison with one-photon imaging, the two-photon image shows much higher imaging contrast for liver tissue of high-fat feeding mice (Figure S21). Besides, 3D one-photon and two-photon fluorescent images along different visual directions were reconstructed by combining the images at different depth (Figure 6). The results indicate that the two-photon imaging could reach a depth of $72 \mu \mathrm{m}$ with superior resolution (Figures 6A and 6B) while the one-photon imaging could only reach a depth of $15 \mu \mathrm{m}$ with lower resolution (Figures 6C and 6D).

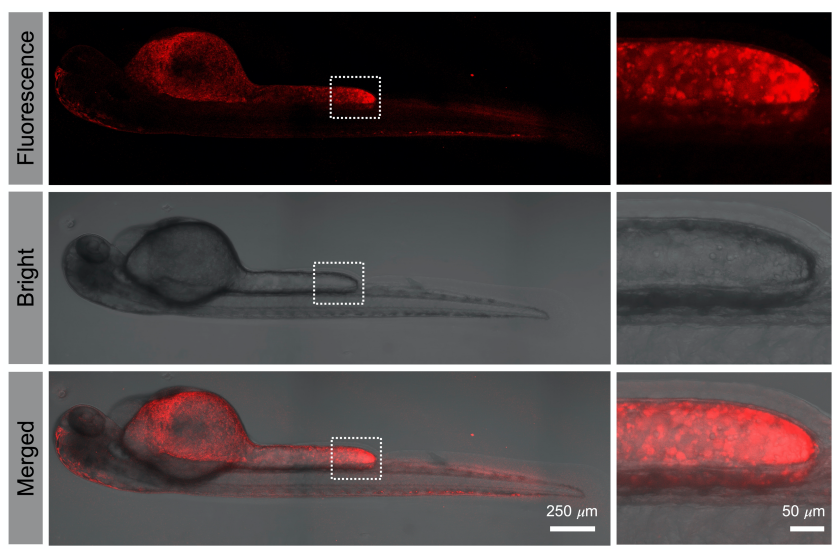

Figure 7. In vivo zebrafish embryo imaging stained with TPABSM (1 $\mu \mathrm{M}, 1 \mathrm{~h}), \lambda_{\mathrm{ex}}=488 \mathrm{~nm}, \lambda_{\mathrm{em}}=550-650 \mathrm{~nm}$. The right is the enlarged part of the white frame in the left.

The excellent performance of TPABSM for LDs imaging suggests it's potential in imaging the LDs in vivo. Yolk sac as a neutral lipid and polar phospholipids storage organ provides energy for the upgrowth of zebrafish. ${ }^{68}$ As shown in Figure 7, intense red fluorescence signals originating from the yolk sac in 3-day-old zebrafish embryos were observed after stained with TPABSM for $30 \mathrm{~min}$. The enlarged parts exhibited more explicit staining images, showing TPABSM was highly specific toward LDs. Therefore, this preliminary result suggests that TPABSM could be used in locating the LDs in the yolk sac, which is essential for observing LDs related biological processes toward in vivo level.

\section{CONCLUSION}

In conclusion, TPABSM, a deep-red emissive AIEgen with bright emission, has been rationally designed and applied for in vitro and in vivo LDs imaging. The conformation lock strategy in solution and solid state has been thoroughly discussed. The results indicate that by rationally integrating the conformation lock effect of intramolecular hydrogen bonding interaction and luminescence wavelength red-shift function of TICT effect, luminogens with DR emission and high quantum yield as well as AIE characteristic could be afforded. As a primary exploration, this work may inspire more investigations on the role of non- covalent interactions in producing high-performance luminescence materials. Besides the bright DR emission, the rational molecular design endows TPABSM with excellent lipophilicity and two-photo absorption characteristic, which enables its application for in vitro and in vivo LDs imaging. As a primary demonstration, the LDs in tissue and the yolk sac of a live fish embryo could be specifically stained and imaged. Further exploration on conformation lock of luminogens through non-covalent interactions currently are under investigation.

\section{ASSOCIATED CONTENT}

\section{Supporting Information}

The Supporting Information is available free of charge on the ACS Publications website.

Experimental details, Photophysical properties, Bio-imaging results (PDF)

\section{AUTHOR INFORMATION}

\section{Corresponding Author}

*To whom correspondence should be addressed:

E-mail: zhaozheng@seu.edu.cn (Z.Z.); tangbenz@ust.hk (T.B.Z.)

\section{Author Contributions}

$\dagger$ These authors contributed equally.

\section{Notes}

The authors declare no competing financial interest.

\section{ACKNOWLEDGMENT}

The authors are grateful for financial support from the National Science Foundation of China (21788102), the Research Grants Council of Hong Kong (N_HKUT609/19, 16305518, A-HKUST605/16, and $\mathrm{C} 6009-17 \mathrm{G}$ ), and the Innovation and Technology Commission (ITC-CNERC14SC01 and ITCPD/17-9).

\section{REFERENCES}

1. Moss, G. P. Basic Terminology of Stereochemistry. Pure \& Appl. Chem. 1996, 68, 2193.

2. Christie, G. H.; Kenner, J. LXXI.-The Molecular Configurations of Polynuclear Aromatic Compounds. Part I. The Resolution of $\gamma$ 6:6'-Dinitro- and 4:6:4':6'-tetranitro-diphenic Acids into Optically Active Components. J. Chem. Soc., Dalton Trans. 1922, 121, 614. 3. Xiong, J. B.; Feng, H. T.; Sun, J. P.; Xie, W. Z.; Yang, D.; Liu, M. H.; Zheng, Y. S. The Fixed Propeller-Like Conformation of Tetraphenylethylene that Reveals Aggregation-Induced Emission Effect, Chiral Recognition, and Enhanced Chiroptical Property. $J$. Am. Chem. Soc. 2016, 138, 11469.

4. Chen, P. Y.; Rassamesard, A.; Chen, H. L.; Chen, S. A. Conformation and Fluorescence Property of Poly(3-hexylthiophene) Isolated Chains Studied by Single Molecule Spectroscopy: Effects of Solvent Quality and Regioregularity. Macromolecules 2013, 46, 5657.

5. Cruz-Cabeza, A. J.; Bernstein, J. Conformation Polymorphism. Chem. Rev. 2014, 114, 2170.

6. Appavoo, S. D.; Huh, S.; Diaz, D. B.; Yudin, A. K. Conformation Control of Macrocycles by Remote Structure Modification. Chem. Rev. 2019, 119, 9724.

7. Lewis, F. D.; Johnson, R. W. Can Molecular Conformation Control Photochemical Behavior? J. Am. Chem. Soc. 1972, 94, 25.

8. Sun, G.; Wei, Y.; Zhang, Z.; Lin, J.; Liu, Z.; Chen, W.; Su, J.; Chou, P.; Tian, H. Diversified Excited - State Relaxation Pathways of Donor - Linker - Acceptor Dyads Controlled by a Bent - to - 
Planar Motion of the Donor. Angew. Chem. Int. Ed. 2020, 59, 18611.

9. Tang, X.; Cui, L. S.; Li, H. C.; Gillett, A. J.; Auras, F.; Qu, Y. K.; Zhong, C.; Jones, S. T. E.; Jiang, Z. Q.; Friend, R. H.; Liao, L. $\mathrm{S}$. Highly efficient luminescence from space-confined chargetransfer emitters. Nat Mater. 2020, DOI: $10.1038 / \mathrm{s} 41563-020$ 0710-z.

10. Wang, T-T.; Xie, G.; Li, H-C.; Yang, S-Y.; Li, H.; Xiao, Y-L.; Zhong, C.; Sarvendra1, K.; Khan1, A.; Jiang, Z.; Liao, L. S. $\pi$ Stacked Thermally Activated Delayed Fluorescence Emitters with Alkyl Chain Modulation. CCS Chemistry 2020, 2, 1757.

11. Wolstenholme, C. H.; Hu, H.; Ye, S.; Funk, B. E.; Jain, D.; Hsiung, C. H.; Ning, G.; Liu, Y.; Li, X.; Zhang, X., AggFluor: Fluorogenic toolbox enables direct visualization of the multi-step protein aggregation process in live cells. J. Am. Chem. Soc. 2020, 142, 17515.

12. Shirbhate, M. E.; Kwon, S.; Song, A.; Kim, S.; Kim, D.; Huang, H. F.; Kim, Y.; Lee, H.; Kim, S. J.; Baik, M. H.; Yoon, J.; Kim, K. M. Optical and Fluorescent Dual Sensing of Aminoalcohols by in Situ Generation of BODIPY-like Chromophore. J. Am. Chem. Soc. 2020, 142, 4975.

13. Liang, J.; Tang, B. Z.; Liu, B. Specific Light-up Bioprobes Based on AIEgen Conjugates. Chem. Soc. Rev. 2015, 44, 2798.

14. Fu, W.; Yan, C. X.; Guo, Z. Q.; Zhang, J. J.; Zhang, H. Y.; Tian, H.; Zhu, W. H., Rational Design of near-infrared aggregation-induced-emission active probes: in situ mapping of annyloidbeta plaques with ultrasensitivity and high-fidelity. J. Am. Chem. Soc. 2019, 141, 3171.

15. He, J. T.; Xu, B.; Chen, F. P.; Xia, H. J.; Li, K. P.; Ye, L.; Tian, W. J. Aggregation-Induced Emission in the Crystals of 9,10-Distyrylanthracene Derivatives: The Essential Role of Restricted Intramolecular Torsion. J. Phys. Chem. C 2009, 113, 9892.

16. Tong, H.; Dong, Y. Q.; Hong, Y. N.; Häussler, M.; Lam, J. W. Y.; Sung, H. H. Y.; Yu, X. M.; Sun, J. X.; Williams, I. D.; Kwok, H. S.; Tang, B. Z. Aggregation-Induced Emission: Effects of Molecular Structure, Solid-State Conformation, and Morphological Packing Arrangement on Light-Emitting Behaviors of Diphenyldibenzofulvene Derivatives. J. Phys. Chem. C 2007, 111, 2287. 17. Li, Y. Y.; Liu, S. J.; Ni, H. W.; Zhang, H. K.; Zhang, H. Q.; Chuah, C.; Ma, C.; Wong, K. S.; Lam, J. W. Y.; Kwok, R. T. K.; Qian, J.; Lu, X. F.; Tang, B. Z. ACQ-to-AIE Transformation: Tuning Molecular Packing by Regioisomerization for Two-Photon NIR Bioimaging. Angew. Chem. Int. Ed. 2020, 59, 12822.

18. Zheng, X. Y.; Peng, Q.; Zhu, L. Z.; Xie, Y. J.; Huang, X. H.; Shuai, Z. G. Unraveling the Aggregation Effect on Amorphous Phase AIE Luminogens: A Computational Study. Nanoscale 2016 , 8,15173

19. Zhao, Z.; Zhang, H. K.; Lam, J. W. Y.; Tang, B. Z. Aggregation-Induced Emission: New Vistas at the Aggregate Level. Angew. Chem. Int. Ed. 2020, 59, 9888.

20. Wang, C.; Qiao, Q.; Chi, W.; Chen, J.; Liu, W.; Tan, D.; McKechnie, S.; Lyu, D.; Jiang, X.; Zhou, W.; Xu, N.; Zhang, Q.; $\mathrm{Xu}, \mathrm{Z}$.; Liu, X. Quantitative Design of Bright Fluorophores and AIEgens via the Accurate Prediction of Twisted Intramolecular Charge Transfer (TICT). Angew. Chem. Int. Ed. 2020, 59, 10160.

21. Kimura, R.; Kuramochi, H.; Liu, P.; Yamakado, T.; Osuka, A.; Tahara, T.; Saito, S. Flapping Peryleneimide as a Fluorogenic Dye with High Photostability and Strong Visible - Light Absorption. Angew. Chem. Int. Ed. 2020, 59, 16430.

22. Zhang, Y. T.; Yan, C. X.; Wang, C.; Guo, Z. Q.; Liu, X. G.; Zhu, W. H. A Sequential Dual-Lock Strategy for Photoactivatable Chemiluminescent Probes Enabling Bright Duplex Optical Imaging. Angew. Chem. Int. Ed. 2020, 132, 9144.

23. Saotome, S.; Suenaga, K.; Tanaka, K.; Chujo, Y. Design for the Enhancement of Environmental Sensitivity in Solid-state Luminescent Boron Complex and Its Multi-step Mechanochromic Property. Mater. Chem. Front. 2020, 4, 1781.
24. Liu, S. J.; Ou, H. L.; Li, Y. Y.; Zhang, H. K.; Liu, J. K.; Lu, X. F.; Kwok, R. T. K.; Lam, J. W. Y.; Ding, D.; Tang, B. Z. Planar and Twisted Molecular Structure Leads to the High Brightness of Semiconducting Polymer Nanoparticles for NIR-IIa Fluorescence Imaging. J. Am. Chem. Soc. 2020, 142, 15146.

25. Grabowski, Z. R.; Rotkiewicz, K.; Rettig, W. Structural Changes Accompanying Intramolecular Electron Transfer: Focus on Twisted Intramolecular Charge-Transfer States and Structures. Chem. Rev. 2003, 103, 3899.

26. Zhao, Z.; Su, H. F.; Zhang, P. F.; Cai, Y. J.; Kwok, R. T. K.; Chen, Y. C.; He, Z. K.; Gu, X. G.; He, X. W.; Sung, H. H. Y.; Willimas, I. D.; Lam, J. W. Y.; Zhang, Z. F.; Tang, B. Z. Polyyne Bridged AIE Luminogens with Red Emission: Design, Synthesis, Properties and Applications. J. Mater. Chem. B 2017, 5, 1650.

27. Lee, W. W. H.; Zhao, Z.; Cai, Y. J.; Xu, Z.; Yu, Y.; Xiong, Y.; Kwok, R. T. K.; Chen, Y.; Leung, N. L. C.; Ma, D. G.; Lam, J. W. Y.; Qin, A. J.; Tang, B. Z. Facile Access to Deep Red/Near-infrared Emissive AIEgens for Efficient Non-doped OLEDs. Chem. Sci. 2018, 9, 6118 .

28. Zhang, G. F.; Chen, Z. Q.; Aldred, M. P.; Hu, Z.; Chen, T.; Huang, Z. L.; Meng, X. G.; Zhu, M. Q. Direct Validation of The Restriction of Intramolecular Rotation Hypothesis via The Synthesis of Novel Ortho-methyl Substituted Tetraphenylethenes and Their Application in Cell Imaging. Chem. Commun. 2014, 50, 12058.

29. Ai, X.; Evans, E.W.; Dong, S; Gillett, A. J.; Guo, H.; Chen, Y.; Hele, T. J. H.; Friend, R. H.; Li, F. Efficient radical-based lightemitting diodes with doublet emission. Nature 2018, 563, 536.

30. Zampetti, A.; Minotto, A.; Cacialli, F. Near-Infrared (NIR) Organic Light-Emitting Diodes (OLEDs): Challenges and Opportunities. Adv. Funct. Mater. 2019, 29, 1807623.

31. Wang, S.; Li, B.; Zhang, F. Molecular Fluorophores for DeepTissue Bioimaging. ACS Cent. Sci. 2020, 6, 1302.

32. Valeur, B. Molecular Fluorecence: Princeples and Applications. Wiley-VCH: Weinheim, 2002.

33. Grabowski, Z. R.; Rotkiewicz, K. Structural Changes Accompanying Intramolecular Electron Transfer: Focus On Twisted Intramolecular Charge-Transfer States and Structures. Chem. Rev. 2003, 103,3899 .

34. Demchenko, A.; Tomin, V. I.; Chou, P.-T. Breaking The Kasha Rule for More Efficient Photochemistry. Chem. Rev. 2017, 117, 13353.

35. Ni, J.-S.; Zhang, X.; Yang, G.; Kang, T.; Lin, X.; Zha, M.; Li, Y.; Wang, L.; Li, K. Angew. Chem. Int. Ed. 2020, 59, 11298.

36. Lee, J.; Kalin, A. J.; Yuan, T.; Al-Hashimi, M.; Fang, L. Fully Conjugated Ladder Polymers. Chem. Sci. 2017, 8, 2503.

37. Chen, W.; Chen, C.-L.; Zhang, Z.; Chen, Y.-A.; Chao, W.-C.; Su, J.; Tian, H.; Chou, P.-T. Snapshotting the Excited-state Planarization of Chemicallly Locked N,N'-disubstituted Dihydrodibenzo[a,c]phenazines. J. Am. Chem. Soc. 2017, 139, 1636.

38. Liu, Y.; Zhang, Z.; Feng, S.; Li, M.; Wu, L.; Hou, R.; Xu, X.; Chen, X.; Bo, Z. Exploiting Noncovalently Conformational Locking as A Design Strategy for High Performace Fused-ring Electron Acceptor Used in Polymer Solar Cells. J. Am. Chem. Soc. 2017, 139,3356

39. Huang, H.; Yang, L.; Facchetti, A.; Marks, T. J. Organic and Polymeric Semiconductors Enhanced by Noncovalent Conformational locks. Chem. Rev. 2017, 117, 10291

40. Sun, T. L.; Kurokawa, T.; Kuroda, S.; Ihsan, A. B.; Akasaki, T.; Sato, K.; Haque, M. A.; Nakajima, T.; Gong, J. P. Physical Hydrogels Composed of Polyampholytes Demonstrate High Toughness and Viscoelasticity. Nat. Mater. 2013, 12, 932.

41. Zhou, H. X.; Pang, X. D. Electrostatic Interactions in Protein Structure, Folding, Binding, and Condensation. Chem. Rev. 2018, $118,1691$. 
42. Gilday, L. C.; Robinson, S. W.; Barendt, T. A.; Langton, M. J.; Mullaney, B. R.; Beer, P. D. Halogen Bonding in Supramolecular Chemistry. Chem. Rev. 2015, 115, 7118.

43. Priimagi, A.; Cavallo, G.; Metrangolo, P.; Resnati, G. The Halogen Bond in the Design of Functional Supramolecular Materials: Recent Advances. Acc. Chem. Res. 2013, 46, 2686.

44. Xia, D. Y.; Wang, P.; Ji, X. F.; Kahashab, N. M.; Sessler, J. L.; Huang, F. H. Functional Supramolecular Polymeric Networks: The Marriage of Covalent Polymers and Macrocycle-Based HostGuest Interactions. Chem. Rev. 2020, 120, 6070.

45. Jing, X.; He, C.; Zhao, L.; Duan, C. Y. Photochemical Properties of Host-Guest Supramolecular Systems with Structurally Confined Metal-Organic Capsules. Acc. Chem. Res. 2019, 52, 100.

46. Qiao, Y. L.; Guo, Y. L.; Yu, C. M.; Zhang, F. J.; Xu W.; Liu, Y. Q.; Zhu, D. B. Diketopyrrolopyrrole-Containing Quinoidal Small Molecules for High-Performance, Air-Stable, and SolutionProcessable n-Channel Organic Field-Effect Transistors. J. Am. Chem. Soc. 2012, 134, 4084.

47. Chen, X.; Addicoat, M.; Jin, E. Q.; Zhai, L. P.; Xu, H.; Huang, N.; Guo, Z. Q.; Liu, L. L.; Irle, S.; Jiang, D. L. Locking Covalent Organic Frameworks with Hydrogen Bonds: General and Remarkable Effects on Crystalline Structure, Physical Properties, and Photochemical Activity. J. Am. Chem. Soc. 2015, 137, 3241.

48. Yan, J. J.; Wang, X. Y.; Wang, M. Z.; Pan, D. H.; Yang, R. L.; Xu, Y. P.; Wang, L. Z.; Yang, M. Self-Assembling Nonconjugated Poly(amide-imide) into Thermoresponsive Nanovesicles with Unexpected Red Fluorescence for Bioimaging. Biomacromolecules 2019, 20, 1455.

49. Zhao, Z.; Zhang, F. J.; Hu, Y. B.; Wang, Z. L.; Leng, B.; Gao, X. K.; Di, C. A.; Zhu, D. B. Naphthalenediimides Fused with 2 (1,3-Dithiol-2- ylidene)acetonitrile: Strong Electron-Deficient Building Blocks for High-Performance n Type Polymeric Semiconductors. ACS Macro Lett. 2014, 3, 1174.

50. Luo, J.; Xie, Z.; Lam, W. Y.; Cheng, L.; Chen, H.; Qiu, C.; Kwok, H. S.; Zhan, X.; Liu, Y.; Zhu, D.; Tang, B. Z. Aggregationinduced Emission of 1-Methyl-1,2,3,4,5-pentaphenylsilole. Chem. Commun. 2001, 1740.

51. Mei, J.; Leung, L. C.; Kwok, T. K.; Lam, W. Y.; Tang, B. Z. Aggregation-induced Emission: Together We Shine, United We Soar! Chem. Rev. 2015, 115, 11718.

52. Johnson, E. R.; Keinan, S.; Mori-Sánchez, P.; Contreras-García, J.; Cohen, A. J.; Yang, W. Revealing Noncovalent Interactions. $J$. Am. Chem. Soc. 2010, 132, 6498.

53. Wu, P.; Chaudret, R.; Hu, X.; Yang, W. Noncovalent Interaction Analysis in Fluctuating Environments. J. Chem. Theory Comput. 2013, 9, 2226.

54. Xing, H.; Li, Z. D.; Wang, W. B.; Liu, J. K.; Song, Y.; Wu, Z. L.; Zhang, W. K.; Huang, F. H. Mechanochemistry of An Interlocked Poly[2]catenane: From Single Molecule to Bulk Gel. CCS Chem. 2019, 1, 513.

55. Xing, H.; Li, Z. T.; Wu, Z. L.; Huang, F. H. Catenane Crosslinked Mechanically Adaptive Polymer Gel. Macromol. Rapid Commun. 2017, 1700361.

56. Guo, B.; Sheng, Z. H.; Hu, D. H.; Liu, C. B.; Zheng, H. R.; Liu, B. Through Scalp and Skull NIR-II Photothermal Therapy of Deep
Orthotopic Brain Tumors with Precise Photoacoustic Imaging Guidance. Adv. Mater. 2018, 30, 1802591.

57. Mao, W. L.; Zhu, M. M.; Yan, C. X.; Ma, Y. Y.; Guo, Z. Q.; Zhu, W. H. Rational Design of Ratiometric Near-Infrared AzaBODIPY-Based Fluorescent Probe for in Vivo Imaging of Endogenous Hydrogen Peroxide. ACS Appl. Bio Mater. 2020, 3, 45.

58. Olzmann, J. A.; Carvalho, P. Dynamics and functions of lipid droplets. Nat. Rev. Mol. Cell. Biol. 2019, 20, 137.

59. Zheng, Z.; Zhang, T.; Liu, H.; Chen, Y.; Kwok, T. K.; Ma, C.; Zhang, P.; Sung, H. Y.; Williams, I. D.; Lam, W. Y.; Wong, K. S.; Tang, B. Z. Bright Near-infrared Aggregation-induced Emission Luminogens with Strong Two-photon Absorption, Excellent Organelle Specificity, and Efficient Photodynamic Therapy Potential. ACS Nano 2018, 12, 8145.

60. Jr. Tian, H.; Sedgwick, A. C.; Han, H. H.; Sen, S.; Chen, G. R.; Zhang, Y.; Sessler, J. L.; James, T. D.; Li J.; He, X. P. Fluorescent Probes for The Imaging of Lipid Droplets In Live Cells. Coord. Chem. Rev. 2021, 427, 213577.

61. An, L.; Xiao, Y. L.; Zhang, S.; Zhang, X. G. Bulky Diamine Ligand Promotes Cross-Coupling of Difluoroalkyl Bromides by Iron Catalysis. Angew. Chem. Int. Ed. 2018, 57, 6921.

62. Zhao, N.; Li, Y.; Yang, W. Y.; Zhuang, J. B.; Li, Y.; Li, N. Multifunctional Pyrazoline Based AIEgens: Real-time Tracking and Specific Protein "Fishing" of Lipid Droplets. Chem. Sci. 2019, 10,9009.

63. Niu, G.; Zhang, R.; Kwong, P. C.; Lam, W. Y.; Chen, C.; Wang, J.; Chen, Y.; Feng, X.; Kwok, T. K.; Sung, H. Y.; Williams, I. D.; Elsegood, M. R. J.; Qu, J.; Ma, C.; Wong, K. S.; Yu, X.; Tang, B. Z. Specific Two-photon Imaging of Live Cellular and Deep-tissue Lipid Droplets by Lipophilic AIEgens At Ultralow Concentration. Chem. Mater. 2018, 30, 4778.

64. Horobin, R. W.; Rashid-Doubell, F.; Pediani, J. D.; Milligan, G. Predicting Small Molecule Fluorescent Probe Localization in Living Cells Using QSAR Dodeling. 1. Overview and Models for Probes of Structure, Properties and Function in Single Cells. Biotech. Histochem. 2013, 88, 440.

65. Kim, H. M.; Cho, B. R. Small-Molecule Two-photon Probes for Bioimaging Applications. Chem. Rev. 2015, 115, 5014.

66. Jiang, M.; Gu, X.; Lam, W. Y.; Zhang, Y.; Kwok, T. K.; Wong, K. S.; Tang, B. Z. Two-photon AIE Bio-probe with Large Stokes Shift for Specific Imaging of Lipid Droplets. Chem. Sci. 2017, 8, 5440 .

67. Sun, X. H.; Zebibula, A.; Dong, X. B.; Zhang, G. X.; Zhang, D. Q.; Qian, J.; He, S. L. Aggregation-Induced Emission Nanoparticles Encapsulated with PEGylated Nano Graphene Oxide and Their Applications in Two-Photon Fluorescence Bioimaging and Photodynamic Therapy in Vitro and in Vivo. ACS Appl. Mater. Interfaces 2018, 10, 25037.

68. Halbach, K.; Ulrich, N.; Goss, K. U.; Seiwert, B.; Wagner, S.; Scholz, S.; Luckenbach, T.; Bauer, C.; Schweiger, N.; Reemtsma, T. Yolk Sac of Zebrafish Embryos as Backpack for Chemicals? Environ. Sci. Technol. 2020, 54, 10159. 
TOC Graphic:

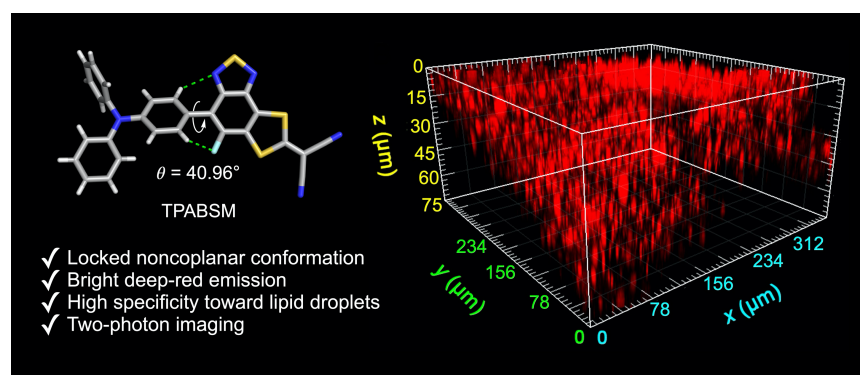

